\title{
Immunohistochemical analysis of brain lesions using S100B and glial fibrillary acidic protein antibodies in arundic acid- (ONO-2506) treated stroke-prone spontaneously hypertensive rats
}

\author{
Hideaki Higashino $\cdot$ Atsuko Niwa $\cdot$ Takao Satou $\cdot$ \\ Yoshio Ohta $\cdot$ Shigeo Hashimoto $\cdot$ Masaki Tabuchi $\cdot$ \\ Kana Ooshima
}

Received: 22 May 2009/Accepted: 17 July 2009/Published online: 6 August 2009

(C) The Author(s) 2009. This article is published with open access at Springerlink.com

\begin{abstract}
Stroke-prone spontaneously hypertensive rats (SHRSP) used as a model of essential hypertension cause a high incidence of brain stroke on the course of hypertension. Incidences and sizes of brain lesions are known to relate to the astrocyte activities. Therefore, relation between brain damage and the expression profile of the astrocytes was investigated with morphometric and immunohistochemical analyses using astrocyte marker antibodies of S100B and glial fibrillary acidic protein (GFAP) with or without arundic acid administration, a suppressor on the activation of astrocytes. Arundic acid extended the average life span of SHRSP. An increase in brain tissue weight was inhibited concomitant with a lower rate of gliosis/hemosiderin deposit/scarring in brain lesions. S100B- or GFAP-positive dot and filamentous structures were decreased in arundic acid-treated SHRSP, and this effect was most pronounced in the cerebral cortex, white matter, and pons, and less so in the hippocampus, diencephalon, midbrain, and cerebellum. Blood pressure decreased after administration of arundic acid in the
\end{abstract}

\footnotetext{
H. Higashino $(\bowtie) \cdot$ A. Niwa $\cdot$ M. Tabuchi $\cdot$ K. Ooshima Department of Pharmacology, Kinki University School of Medicine, Osaka-Sayama, Osaka 589-8511, Japan e-mail: higasino@med.kindai.ac.jp

T. Satou

Department of Pathology, Kinki University School of Medicine,

Osaka-Sayama 589-8511, Japan

Y. Ohta

Department of Clinical Examinations, Kinki University

Nara Hospital, Nara 630-0293, Japan

S. Hashimoto

Department of Clinical Pathology, PL Hospital,

Osaka 584-8585, Japan
}

high-dose group $(100 \mathrm{mg} / \mathrm{kg} /$ day arundic acid), but not in the low-dose group $(30 \mathrm{mg} / \mathrm{kg} / \mathrm{day})$. These data indicate that arundic acid can prevent hypertension-induced stroke, and may inhibit the enlargement of the stroke lesion by preventing the inflammatory changes caused by overproduction of the S100B protein in the astrocytes.

Keywords Arundic acid · Astrocyte - Brain - GFAP . S100B $\cdot$ SHRSP

\section{Introduction}

Stroke-prone spontaneously hypertensive rats (SHRSP) (Okamoto et al. 1974) developed from SHR (Okamoto and Aoki 1963) by the selective crossbreeding are widely used for the investigations of hypertension and stroke as a model of human essential hypertension, which cause a high incidence of brain stroke more than $90 \%$ concomitant with myocardial fibrosis and arterionecrosis in the kidney during stage of severe hypertension. S100B and glial fibrillary acidic protein (GFAP) are considered to be relatively astrocyte-specific and astrocyte-specific marker proteins, respectively (Steiner et al. 2007). S100B specificity varies between animal species (Boyes et al. 1986). This protein is an acidic calcium-binding protein that is produced mainly by astrocytes. Depending on its concentration, S100B has two opposing effects, trophic and toxic (Rothermundt et al. 2003). At nanomolar concentrations, S100B stimulates neurite outgrowth and enhances survival of neurons. However, at micromolar concentrations, S100B stimulates the expression of proinflammatory cytokines such as IL-6 and induces apoptosis. S100B protein, previously called $\mathrm{S} 100 \beta$ protein, exists as a $\beta \beta$ dimer in the cytoplasm of astrocytes (Boyes et al. 1986). GFAP is an intermediate 
filament protein found in reactive astrocytes (Reymond et al. 1996; Ridet et al. 1996). The roles and precise localization of each protein are not fully understood.

Up-regulation of S100B protein synthesis and leakage of S100B from damaged astrocytes that express GFAP in the glial scar can be induced by transient middle cerebral artery occlusion in rats. These events lead to an increase in the infarct volume (Matsui et al. 2002; Yasuda et al. 2004). The Ono Pharmaceutical Co. Ltd (Osaka, Japan) has discovered that arundic acid ((2R)-2-propyloctanoic acid, ONO-2506) is a potent inhibitor of the production and release of S100B protein from astrocytes (Shimoda et al. 1998). During development of this drug, it was discovered that administration of arundic acid to experimental animals reduced the expression of $\mathrm{S} 100 \mathrm{~B}$ protein and GFAP in activated astrocytes, and inhibited the increase in the infarct volume of brain injured animals (Tateishi et al. 2002; Mori et al. 2004).

Since brain injuries such as cerebral hemorrhage, infarction, and subarachnoidal hemorrhage spontaneously occur with high frequency in stroke-prone spontaneously hypertensive rats (SHRSP) during the advanced stages of hypertension we used these animals as a hypertensioninducing brain lesion model. Our study examined the effects of arundic acid on brain damage and the profiles of S100B and GFAP, expressed by astrocytes. We used morphometry and immunohistochemistry to examine brain lesions on the course of hypertension.

\section{Materials and methods}

\section{Animals and experimental design}

Male SHRSP (Okamoto et al. 1974) and Wistar Kyoto rats (WKY) aged 6 weeks and with normal blood pressure were purchased from Kinki University Animal Center (Osaka, Japan) and maintained under lighting control (light and dark phases for $12 \mathrm{~h}$ each) at a temperature of $15 \pm 2{ }^{\circ} \mathrm{C}$ and in $60 \%$ humidity. Arundic acid was provided by Ono Pharmaceutical Co. Ltd (Osaka, Japan) as a candidate for suppression of the activation of astrocytes (Tateishi et al. 2002). Three SHRSP groups of rats ( $n=6$ each) were studied. Each group initially consisted of ten rats to survey the process of cerebral lesions. Brains were obtained from one rat in each group every 4 weeks for 12 weeks by infusion of $4 \%$ paraformaldehyde buffer solution ( $\mathrm{pH} 7.0-$ 7.5) into the cerebral artery under sodium pentobarbital anesthesia. After the 12th week of the experiment, there were no significant morphological findings in the brains of rats in any group, and so the remaining six rats in each group were morphologically investigated after that. No arundic acid (control), $30 \mathrm{mg} / \mathrm{kg} /$ day arundic acid (low dose) mixed with SP-2 chow (Funabashi Farm, Chiba, Japan), and $100 \mathrm{mg} / \mathrm{kg} /$ day arundic acid (high dose) mixed with SP-2 chow were given to three groups of rats, respectively. Experiments were continued until 61 weeks of age, at which time animals were killed if they had not already died. Doses were determined by Ono Pharmaceutical Co. Ltds information that dosages of 3, 10, and $30 \mathrm{mg} / \mathrm{kg}$ p.o. prevented S100B release into the cerebral spinal fluid of rats during experimental cerebral ischemia. WKY rats were fed with SP-2 chow without arundic acid. Tap water was available ad libitum. Once a week, all rats were weighed and their systolic arterial blood pressure was measured with the tail-cuff method using a photoelectric detector (UR-5000, Ueda, Tokyo, Japan). The brains from rats that died spontaneously during the experimental period and from rats that were killed with euthanasia using an overdose of sodium pentobarbital $(50 \mathrm{mg} / \mathrm{kg}$ i.p.) at the 61 st week were collected for analysis. To avoid postmortem organ changes, brains were removed as soon as possible after discovery of a dead animal during the regular rounds that occurred four times a day. All animals used in this experiment were handled with due care according to the guidelines established by the Japanese Association for Laboratory Animal Science, which complies with international rules and policies.

\section{Morphometric and immunohistochemical analyses}

The brains were carefully resected and post-fixed in $4 \%$ paraformaldehyde solution in $0.1 \mathrm{M}$ phosphate-buffer (PB, $\mathrm{pH}$ 7.0-7.5) for $12 \mathrm{~h}$, and then rinsed with PB. After fixed tissues were embedded in paraffin wax, 3- $\mu$ m-thick serial coronal sections were obtained and mounted on poly-Llysine-coated glass slides. For morphometric analysis, hematoxylin-eosin staining was performed according to standard protocols. For immunohistochemical analysis, paraffin-embedded sections were dewaxed in xylene and hydrated. S100B antigen was retrieved by heating sections in $10 \mathrm{mM}$ sodium citrate buffer ( $\mathrm{pH}$ 6.0) in a microwave oven for $20 \mathrm{~min}$, and sections were then cooled in de-ionized water. For GFAP staining, the sections were incubated in buffer solution containing $0.1 \%$ trypsin for $5 \mathrm{~min}$ at room temperature. Endogenous peroxidase activity was quenched by incubation of the sections for 5 min with $3 \%$ hydrogen peroxide. Sections were incubated overnight at $4^{\circ} \mathrm{C}$ in primary antibodies for $\mathrm{S} 100$ rabbit polyclonal antibody (Code No. Z 0311; 1:800; DAKO, Glostrup, Denmark), or GFAP rabbit polyclonal antibody (Code No. Z 0334; 1:500; DAKO). The specificity of the S100B antibody was determined by western blotting of purified human recombinant S100 protein (Ilg et al. 1996). The antibody labels S100B strongly, S100A1 weakly, and S100A6 very weakly. The anti-GFAP 
antibody does not cross-react in immunocytochemical assays with vimentin or other intermediate filament proteins, as described in a previous report (Rettig et al. 1986). Staining was detected using biotin-labeled anti-rabbit secondary antibodies and streptavidin conjugated to horseradish peroxidase (DAKO LSAB2 System; DAKO Cytomation, Carpinteria, CA, USA), according to the manufacturer's instructions. Reaction products were visualized using diaminobenzidine (Sigma-Aldrich Co., St. Louis, MO, USA). Nuclei were stained with hematoxylin. Rabbit immunoglobulin (DAKO, Glostrup, Denmark) and secondary antibodies were used for negative controls. Sections were examined with microscope (ECLIPSE E800; Nikon, Tokyo, Japan) using computer-assisted image analysis software (Mac Scope Version 2.5 software; Mitani Co., Fukui, Japan). The areas of the tissue examined by microscope were $136 \mu \mathrm{m} \times 96 \mu \mathrm{m} \quad(696 \times 492$ pixels $)=1.3 \times 10^{4} \mu^{2} \quad\left(3.42 \times 10^{5}\right.$ pixels $)$ for $\mathrm{S} 100 \mathrm{~B}$ investigation, and $200 \mu \mathrm{m} \times 150 \mu \mathrm{m}(1,024 \times 768$ pixels $)=3.0 \times 10^{4} \mu^{2} \quad\left(7.86 \times 10^{5}\right.$ pixels $)$ for GFAP investigation. Depending on the size of the area, four to five areas from each specimen were observed by microscope. Average values were calculated using the data obtained from 22-42 areas in SHRSP, and 15-21 areas in WKY. Color-stained dots greater than 10 pixels $\left(0.38 \mu \mathrm{m}^{2}\right)$ that were associated with antibody staining were counted as the least fragment of astrocytes, and the total dot area in the specimen was calculated using computer-assisted image analysis software, and compared among the four groups of rats.

\section{Statistical analysis}

All results were expressed as the mean \pm SEM. Comparisons between means of groups were made using a one-way analysis of variance (ANOVA) and a Scheffe multiple comparisons test, or a two-way ANOVA test for the comparison between S100B or GFAP immunostaining specimens. Survival was analyzed using standard KaplanMeier analysis with a Mantel-Cox log-rank test. For all tests, differences were considered statistically significant at a value of $p<0.05$.

\section{Results}

Effects of arundic acid on physical indices

The blood pressure levels of the three SHRSP groups were the same at the beginning of the experiment. In the highdose group (100 mg/kg/day arundic acid), the rise in blood pressure was significantly decreased after the eighth week until around the 36th week of the experiment. There were

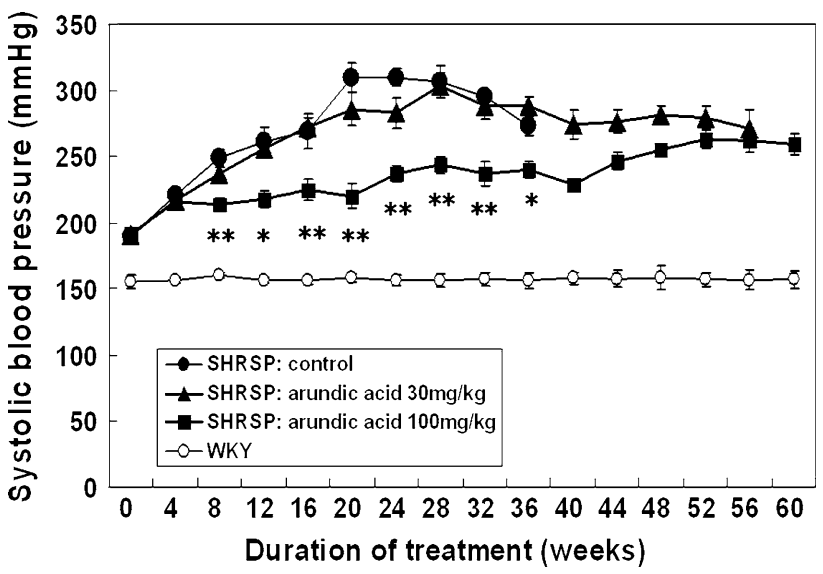

Fig. 1 Effects of arundic acid on blood pressure in SHRSP and WKY rats. Blood pressure was measured by the tail-cuff method. Each group consisted of six rats. Filled circle SHRSP: control, filled triangle SHRSP: $30 \mathrm{mg} / \mathrm{kg} /$ day arundic acid, filled square SHRSP: $100 \mathrm{mg} / \mathrm{kg} / \mathrm{day}$ arundic acid, open circle WKY. * and **indicate significant differences of $p<0.05$ and $p<0.01$, respectively, compared with control SHRSP

no differences in the blood pressure of the low dose (30 mg/kg/day arundic acid) or control groups of SHRSP from the beginning of the experiment until their death at week 36 (Fig. 1). No significant differences were noted in body weight or heart rate during the experiment among the SHRSP groups as follows: for example, at 24 weeks when all rats were still alive and in good condition, body weight was $224.5 \pm 2.7 \mathrm{~g}$ in the SHRSP control group, $224.0 \pm$ $3.4 \mathrm{~g}$ in the SHRSP low-dose group, and $234.2 \pm 2.7 \mathrm{~g}$ in the SHRSP high-dose group. Heart rate at this time was $451 \pm 6 \mathrm{bpm}$ in the SHRSP control group, $410 \pm 5 \mathrm{bpm}$

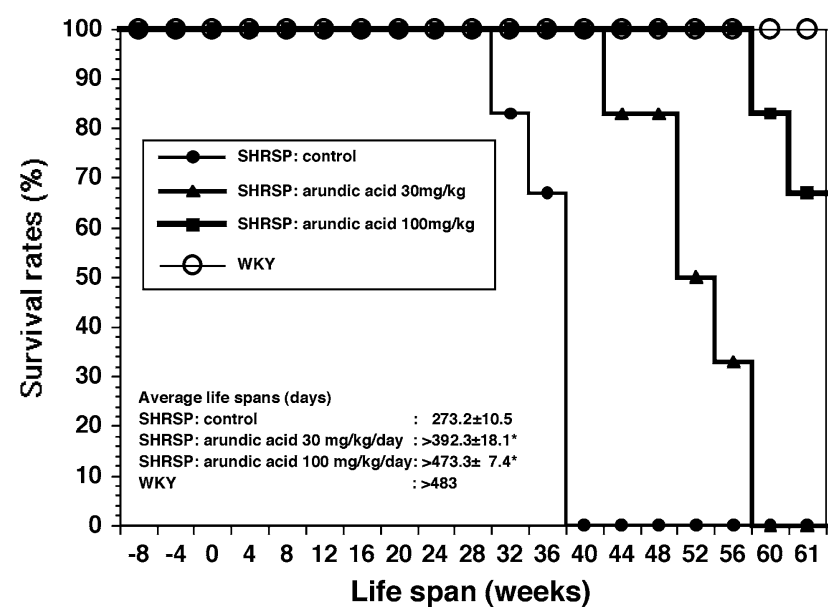

Fig. 2 Comparison of survival rates in the SHRSP and WKY groups with or without oral administration of arundic acid. Each group consisted of six rats. Time 0 is the start point of the experiments. Filled circle SHRSP: control, filled triangle SHRSP: $30 \mathrm{mg} / \mathrm{kg} / \mathrm{day}$ arundic acid, filled square SHRSP: $100 \mathrm{mg} / \mathrm{kg} / \mathrm{day}$ arundic acid, filled circle WKY. *indicates significant difference of $p<0.05$ compared with control SHRSP 
Table 1 Cerebral abnormal findings at the time of natural death or killing at the age of 69 weeks in SHRSP and WKY, and average lifespans with or without arundic acid medication

\begin{tabular}{llc}
\hline Rats/treatments & Cerebral findings at autopsy in six cases & Average life span (days) \\
\hline SHRSP/control $(n=6)$ & Five cases of cerebral thrombosis, and one case of hemorrhage & $273 \pm 10.5$ \\
SHRSP/30 mg/kg/day & Three cases of cerebral thrombosis, and one case of subarachnoidal & $392.3 \pm 18.1^{*}$ \\
arundic acid $(n=6)$ & hemorrhage $($ cf. three cases of heart failures with edema) & \\
SHRSP/100 $\mathrm{mg} / \mathrm{kg} /$ day & One case of cerebral thrombosis, and two cases of subarachnoidal & $>473.3 \pm 7.4^{*}$ \\
arundic acid $(n=6)$ & hemorrhage (cf. $3 \times$ survives) & $>483^{*}$ \\
WKY & No brain lesion (cf. six cases of survives) & \\
\hline
\end{tabular}

* Represents the significant differences at the level of $p<0.05$ compared with SHRSP control group

in the SHRSP low-dose group, and $418 \pm 5 \mathrm{bpm}$ in the SHRSP high-dose group.

Survival rates and average life spans of the SHRSP groups

The survival rate in the control SHRSP group rapidly declined after the 30th week, and the average life span $(273.2 \pm 10.5$ days) was the shortest among the groups (Fig. 2). The low-dose group showed an upper shift in the survival rate, and the average life span $(>392.3 \pm$ 18.1 days) was longer compared to control. The survival rate of the high-dose group showed a strong upper shift compared with the low-dose group, and the average life span $(>473.3 \pm 7.4$ days) was much longer compared with the control and low-dose groups. WKY rats given no arundic acid lived more than 483 days, longer than the end of the experimental period (Table 1).

Cerebral morphometric findings, incidences of morphological changes, and brain wet weights at the time of natural death or killing at the 61 st week in SHRSP groups

Cerebral autopsy was done at the time of natural death or killing at the 61st week in SHRSP (Table 1). The SHRSP control group showed five cerebral thromboses and one hemorrhage in a total of six rats. On the other hand, the low- and high-dose groups showed three cerebral thromboses, one subarachnoidal hemorrhage, and three heart failures in a total of six rats, and one cerebral thrombosis, two subarachnoidal hemorrhages, and three survivors at 61 weeks in the six total rats, respectively. On the other hand, neither death nor brain lesions were noted in WKY animals. As shown in Table 2, brain softening, bleeding, angionecrosis, and gliosis/hemosiderin deposit/scarring changes were observed in $5 / 6,2 / 6,5 / 6$, and $6 / 6$ control SHRSP, respectively. Thus, these lesions occurred in almost all rats. All abnormal morphological changes were decreased by the administration of arundic acid as shown in Fig. 3. In particular, gliosis/hemosiderin deposit/scarring changes were completely eliminated in high-dose animals $(p<0.05$ vs. control SHRSP). No abnormal morphological changes were found in WKY rats. Brain tissue extending from the cerebrum to the medulla oblongata was isolated from the skull and weighed immediately after autopsy (Table 3). In the low- and high-dose groups, the brain tissue weight and the ratio of brain/body weight were significantly decreased compared with control SHRSP, and were similar to that seen in WKY rats. However, these preferable effects caused by the administration of arundic acid were not observed in the heart and kidney (Fig. 3).

Table 2 Incidences of morphological changes in the brain at the time of natural death or killing at the age of 69 weeks in SHRSP with or without arundic acid medication

\begin{tabular}{lllll}
\hline Rats/treatments & \multicolumn{2}{l}{ Morphological findings } & \\
\cline { 2 - 5 } & Softening & Bleeding & Angionecrosis & $\begin{array}{c}\text { Gliosis/hemosiderin } \\
\text { deposit/scaring }\end{array}$ \\
\hline SHRSP/control & $5 / 6$ & $2 / 6$ & $5 / 6$ & $6 / 6$ \\
SHRSP/30 mg/kg/day arundic acid & $2 / 6$ & $1 / 6$ & $1 / 6$ & $2 / 6$ \\
SHRSP/100 mg/kg/day arundic acid & $2 / 6$ & $1 / 6$ & $2 / 6$ & $0 / 6^{* \mathrm{c}}$ \\
WKY & $0 / 6^{* \mathrm{a}}$ & $0 / 6$ & $0 / 6^{* \mathrm{~b}}$ & $0 / 6^{* \mathrm{c}}$ \\
\hline
\end{tabular}

$*^{\mathrm{a}}, *^{\mathrm{b}}$, and $*^{\mathrm{c}}$ represent the significant differences at the level of $p<0.05$ compared with each parameter of SHRSP control group 

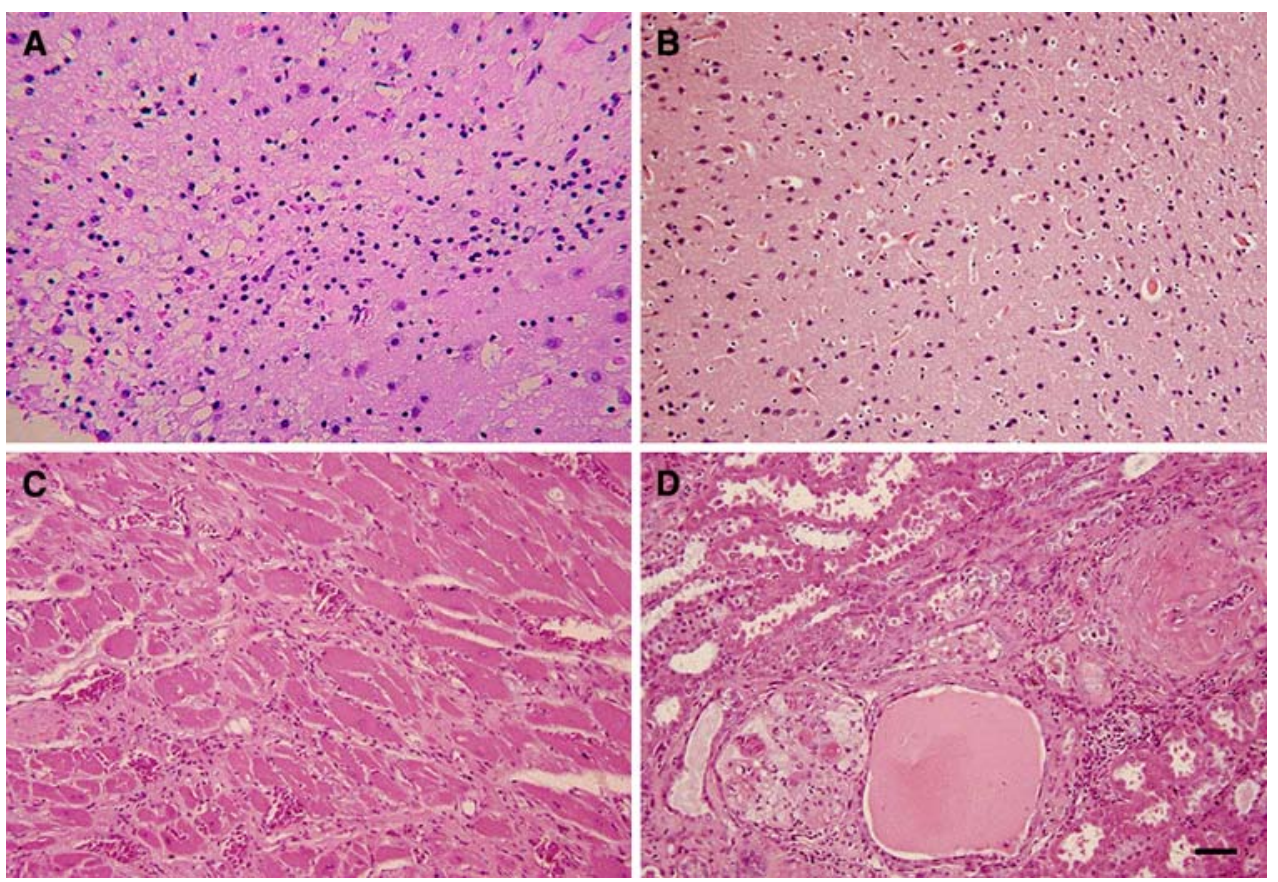

Fig. 3 Histological findings of brain, heart, and kidney tissues stained by hematoxylin-eosin (HE) in SHRSP. a Cerebrovascular lesions (softening, hemorrhage) in the cortex of the brain were seen in untreated SHRP. Findings of gliosis/hemosiderin deposit/scaring were often observed in the cortex and/or white matter close to the stroke lesions (not shown here). b No cerebrovascular lesions were found in

arundic acid-treated SHRSP, especially in high doses. c Myocardial necrosis and fibrosis in the surrounding myocardium were noted in arundic acid-treated SHRSP. d Angionecrosis, collapse of the glomerulus were noted, and distal and proximal tubules were enlarged by containing proteinuria and their epithelium was atrophic and flattened in arundic acid-treated SHRSP. Scale bar equals $50 \mu \mathrm{m}$

Table 3 Comparison of brain and body weights, and rates of brain/body weight (\%) at the autopsy in WKY and SHRSP groups

\begin{tabular}{llll}
\hline Rats/treatments & Brain weights $(\mathrm{g})$ & Body weights $(\mathrm{g})$ & Brain/body weights $(\%)$ \\
\hline SHRSP/control & $3.33 \pm 0.21$ & $244.3 \pm 16.9$ & $1.40 \pm 0.12$ \\
SHRSP/30 mg/kg/day arundic acid & $2.47 \pm 0.04^{* \mathrm{a}}$ & $325.0 \pm 36.4^{* \mathrm{~b}}$ & $0.81 \pm 0.90^{* \mathrm{c}}$ \\
SHRSP/100 mg/kg/day arundic acid & $2.53 \pm 0.11^{\mathrm{a}}$ & $322.2 \pm 8.8^{* \mathrm{~b}}$ & $0.79 \pm 0.05^{* \mathrm{c}}$ \\
WKY & $2.11 \pm 0.08^{* \mathrm{a}}$ & $436.4 \pm 3.5^{* \mathrm{~b}}$ & $0.48 \pm 0.01 *^{\mathrm{c}}$ \\
\hline
\end{tabular}

$*^{\mathrm{a}}, *^{\mathrm{b}}$, and ${ }^{* \mathrm{c}}$ represent the significant differences at the level of $p<0.001$ compared with each parameter of SHRSP control group

\section{S100B and GFAP immunohistochemical analyses}

\section{Cerebral cortex, white matter, and hippocampus}

In the cerebral cortex and white matter of control SHRSP, S100B antibody-reactive dot and filamentous structures (panels b in Figs. 4, 5) were diffusedly distributed, and the sum of the areas of the particles was markedly increased or tended to increase compared with WKY brains (panels a in Figs. 4, 5). Although the total area of dot and filamentous structures was less in the low-dose SHRSP group compared with control in the cortex, no difference was observed between the low- and high-dose groups of SHRSP (panels c, d in Fig. 4; Fig. 6 top left). In the white matter, the administration of arundic acid inhibited the expression of the particles in a dose-dependent manner (panels c, d in Fig. 5; Fig. 6 row 2, left). The shapes of the dots immunostained by the S100B antibody were consistent with astrocytic morphology in both of the tissues. In addition, many round particles, small dots, and circles that appeared to be blood vessels were also observed. The sum of the area occupied by the dots encompassed more than $15 \%$ of the total specimen area in the cortex. GFAPreactive dots and filamentous structures in the cerebral cortex and white matter of SHRSP were markedly increased compared with WKY brains, and the abundance of these GFAP-reactive structures was reduced by arundic acid in a dose-dependent manner (panels e-h in Fig. 4; Fig. 6 top right; panels e-h in Fig. 5; Fig. 6 row 2, right). The shapes of the dots immunostained by the GFAP antibody showed spider-like astrocytic morphology in both of the tissues. 
Fig. 4 Representative photographs of cerebral cortex immunostained by S100B and GFAP antibodies and hematoxylin staining in WKY rats and SHRSP with or without administration of arundic acid. a-d Representative immunostaining of sections stained with the S100B antibody from the following groups: WKY, SHRSP: control, SHRSP: $30 \mathrm{mg} / \mathrm{kg} /$ day arundic acid, and SHRSP: $100 \mathrm{mg} / \mathrm{kg} /$ day arundic acid, respectively. e-h Representative

immunostaining of sections stained with the GFAP antibody from the following groups: WKY, SHRSP: control, SHRSP: $30 \mathrm{mg} / \mathrm{kg} / \mathrm{day}$ arundic acid, and SHRSP: $100 \mathrm{mg} / \mathrm{kg} /$ day arundic acid, respectively. Scale bar equals $50 \mu \mathrm{m}$

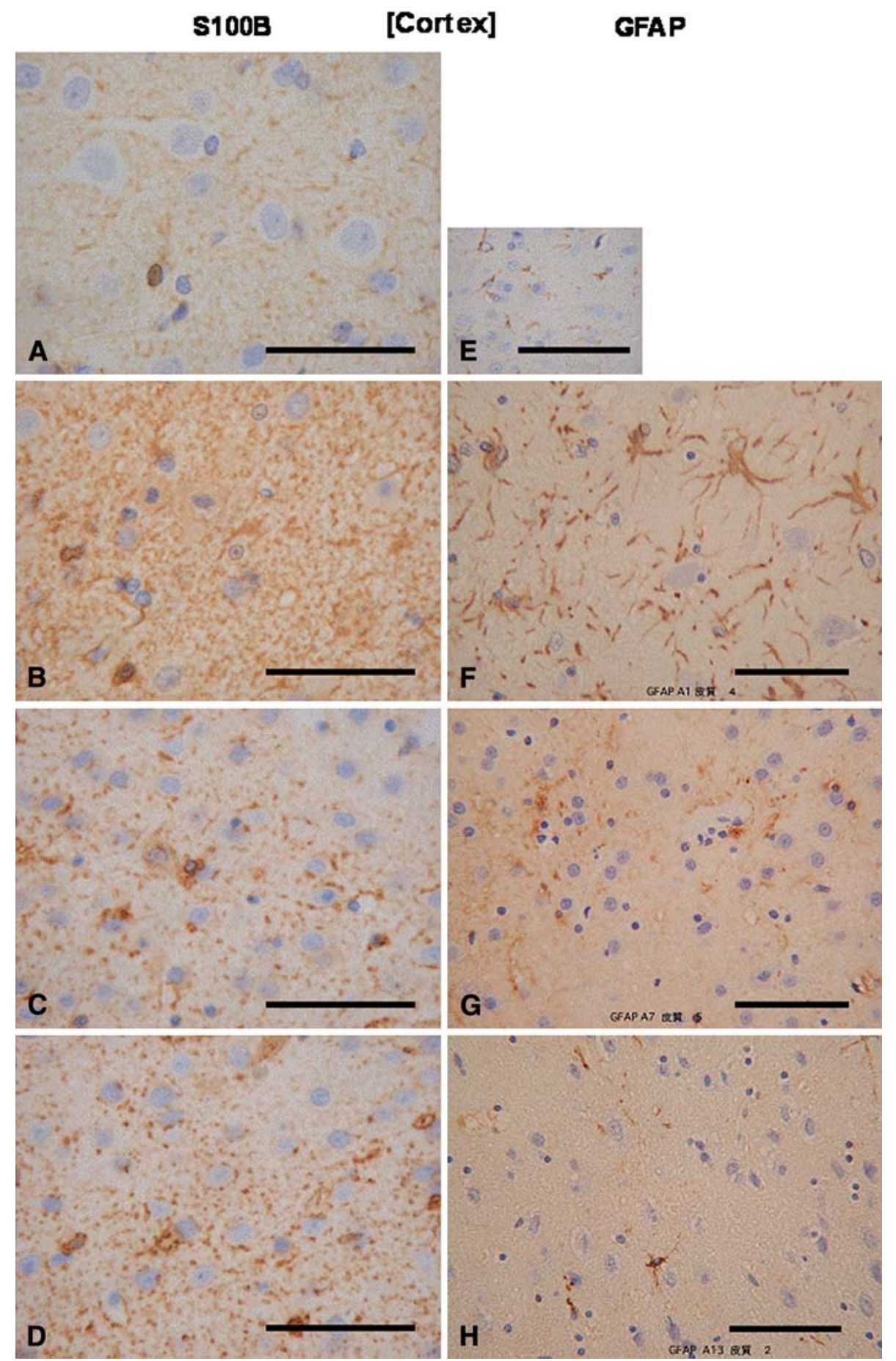

In the hippocampus, however, S100B antibody-reactive dot and filamentous structures showed no difference between WKY brains and control SHRSP. In the SHRSP groups, administration of arundic acid did not induce a change in the area occupied by dot and filamentous structures (Fig. 6 row 3, left). The shapes of the dots immunostained by the S100B antibody were consistent with astrocytic morphology. Curved lines, small and large dots, and circles that appeared to be blood vessels were also observed. Structures consistent with astrocyte-like morphology appeared to be reduced compared to other irregularly shaped structures (data not shown). The area occupied by GFAP antibody-reactive particles in the hippocampus in control SHRSP was not different from those in WKY, and scattered astrocyte-like morphology was occasionally. Only SHRSP given a high dose of arundic acid showed an inhibition in the sum of the areas occupied by GFAP-positive astrocytes (Fig. 6 row 3, right. Data not shown). 
Fig. 5 Representative photographs of cerebral white matter immunostained by S100B and GFAP antibodies and hematoxylin staining in WKY rats and SHRSP with or without administration of arundic acid. a-d

Representative immunostaining of sections stained with the S100B antibody from the following groups: WKY, SHRSP: control, SHRSP: $30 \mathrm{mg} / \mathrm{kg} /$ day arundic acid, and SHRSP: $100 \mathrm{mg} / \mathrm{kg} /$ day arundic acid, respectively. $\mathbf{e}-\mathbf{h}$

Representative immunostaining of sections stained with the GFAP antibody from the following groups: WKY, SHRSP: control, SHRSP: $30 \mathrm{mg} / \mathrm{kg} /$ day arundic acid, and SHRSP: $100 \mathrm{mg} / \mathrm{kg} /$ day arundic acid, respectively. Scale bar equals $50 \mu \mathrm{m}$

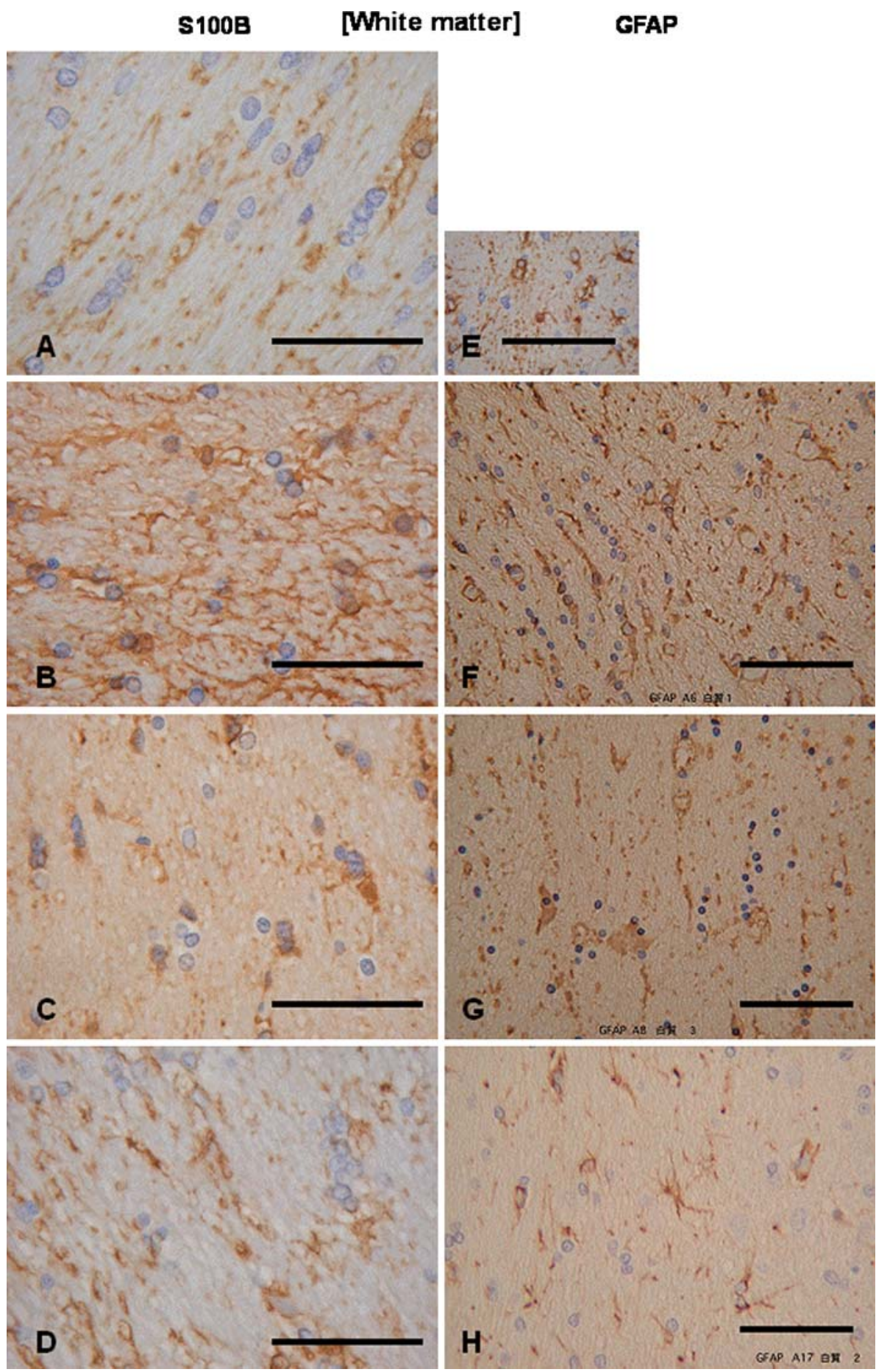

\section{Pons, diencephalons, midbrain and cerebellum}

In the pons, the total area occupied by S100B antibodyreactive dot and filamentous structures in control SHRSP was markedly increased compared with WKY brains (Fig. 6 row 4, left). The shapes of the dots immunostained by the S100B antibody were consistent with astrocytic morphology. Many types of curved lines, small and large dots, and circles that appeared to be blood vessels were also observed, resembling those in the hippocampus. This increase was suppressed by the administration of arundic acid in a dose-dependent manner in SHRSP (Fig. 6 row 4, left. Data not shown). The sum of the area occupied by GFAP antibody-reactive dot and filamentous structures 
Fig. 6 Sum of the areas of particles immunostained by the S100B (left side) and GFAP (right side) antibodies in the cortex, white matter,

hippocampus, and pons in WKY rats and SHRSP without and with arundic acid

administration. White columns WKY $(n=6)$, Black columns SHRSP: control, $30 \mathrm{mg} / \mathrm{kg} / \mathrm{day}$ arundic acid, and $100 \mathrm{mg} / \mathrm{kg} /$ day arundic acid ( $n=6$ each). From top to bottom cortex, white matter, hippocampus, and pons Comparisons between the groups were adopted a two-way ANOVA test. $*$ and $* * p<0.05$ and $P<0.001$ versus WKY, $\dagger$ and $\dagger \dagger p<0.05$ and $p<0.001$ versus. SHRSP control, and and $\uparrow p<0.05$ and $p<0.001$ versus SHRSP $30 \mathrm{mg} / \mathrm{kg}$, respectively
S100B
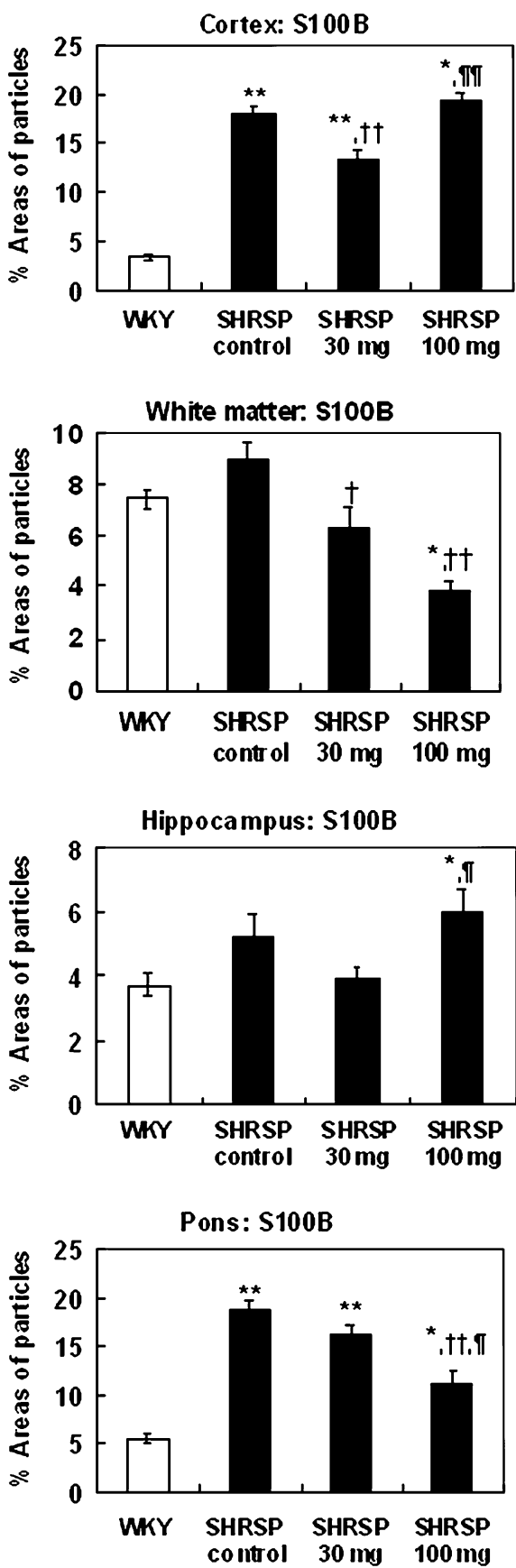

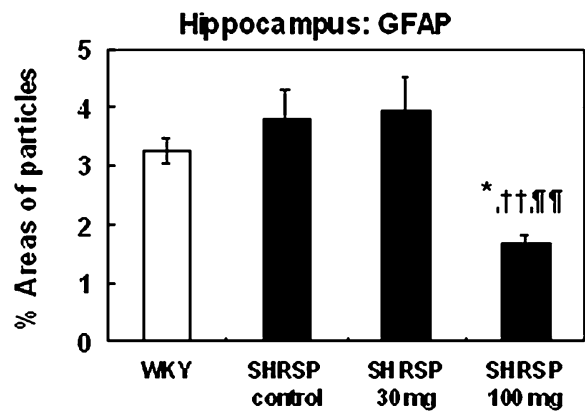

GFAP
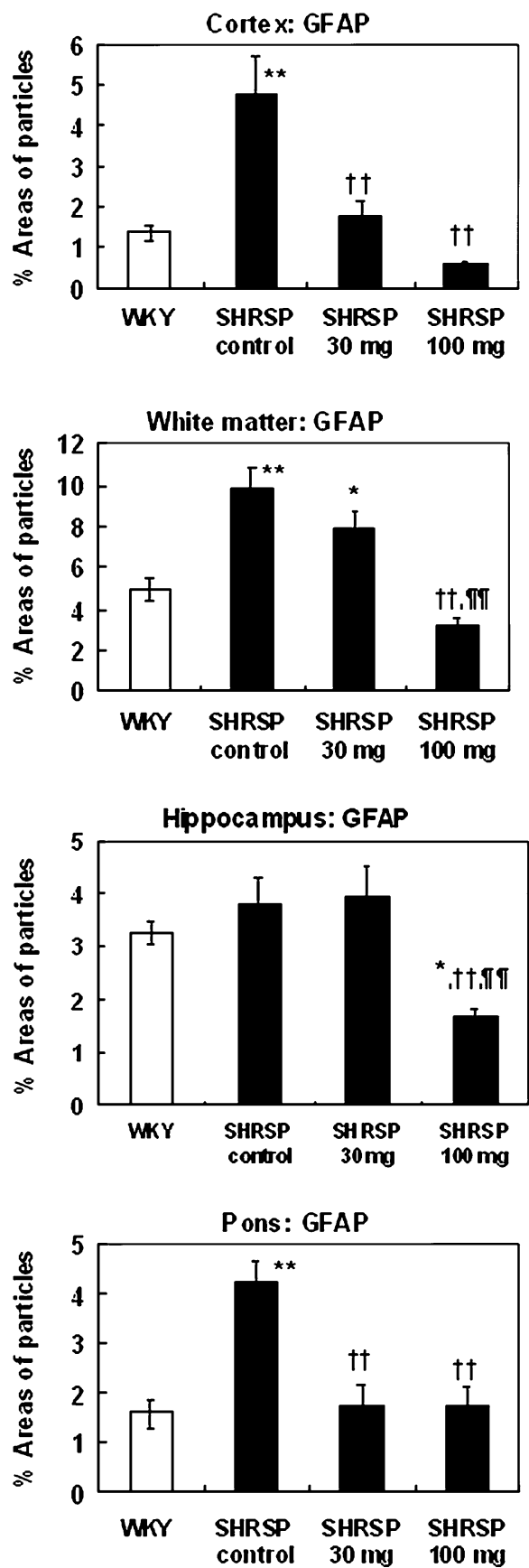

with astrocytic morphology was increased in control SHRSP compared with WKY brains, and was decreased in the brains of SHRSP given both low and high doses of arundic acid (Fig. 6 row 4, right. Data not shown).

In the diencephalons, the sum of the area occupied by GFAP antibody-reactive dots was increased in SHRSP and was decreased by both doses of arundic acid. Conversely, the area occupied by S100B antibody-reactive dots was not decreased by the administration of arundic acid (data not shown).
In the midbrain and cerebellum of SHRSP, the S100B antibody-reactive dot and filamentous structures were markedly increased compared with WKY brains, and arundic acid inhibited the increase in S100B-positive structures (data not shown). The sum of the area occupied by GFAP antibody-reactive dot and filamentous structures was not significantly affected by arundic acid, although the sum of the areas of the particles was higher in control SHRSP than in WKY brains (data not shown). 


\section{Discussion}

SHRSP is a widely accepted disease model for human hypertension and stroke because the spontaneous severe hypertension leads to a high incidence of brain injuries (Kataoka et al. 1994; Steiner et al. 2007). In SHRSP, blood pressure elevates with age from around $180 \mathrm{mmHg}$ at 8 weeks of age to around $300 \mathrm{mmHg}$ at 30 weeks of age. In almost all rats older than 34 weeks of age, the strokes leading to cerebral bleeding, thrombosis, and subarachnoidal hemorrhage occur spontaneously.

Arundic acid was developed as an agent that inhibits the synthesis of S100B in astrocytes (Shimoda et al. 1998), and has been found to significantly decrease the mRNA expressions of S100B, NGF- $\beta$, inducible nitric oxide synthase (iNOS), and cyclooxygenase 2 (COX-2) (Shimoda et al. 1998; Tateishi et al. 2002; Shinagawa et al. 2006). In this experiment, the effects of arundic acid on stroke caused by severe hypertension were studied by examining S100B and GFAP expression in cerebral lesions.

Long-term oral administration of arundic acid to SHRSP provided several benefits. Arundic acid conferred marked longevity and decreases in cerebral lesions such as thrombosis and hemorrhages, compared to control SHRSP (Tables 1, 2). The blood pressure level was significantly decreased in the high-dose SHRSP group but not in the low-dose SHRSP group. Hypotensive effects of arundic acid at a high dose have been reported in general pharmacological studies performed by Ono Pharmaceutical Co. Ltd. They have shown that acute administration of $300 \mathrm{mg} /$ $\mathrm{kg}$ p.o. arundic acid to rats decreased blood pressure by $9 \%$ without affecting the heart rate and that administration of $600 \mathrm{mg} / \mathrm{kg}$ decreased blood pressure by $18 \%$ concomitant with a $25 \%$ decrease in the heart rate. We also obtained an acute hypotensive effect when rats were given an intravenous injection of arundic acid at more than $10 \mathrm{mg} / \mathrm{kg}$. This was due to $\alpha$ blocking activity, which was suggested by results from isolated hearts treated with arundic acid using a Langendorf apparatus (unpublished data). In our study, chronic $100 \mathrm{mg} / \mathrm{kg}$ p.o. administration of arundic acid suppressed the elevation of blood pressure by the eighth week of the experiment. The occurrence of stroke was less in the high-dose arundic acid group compared to what was observed in the low-dose group, which is indicative of a protective effect due to the hypotensive properties of the drug. Chronic administration of arundic acid to SHRSP prevented the occurrence of stroke and brain edema (Tables 1, 2, 3) at both low and high doses, and pathological changes such as gliosis/hemosiderin deposit/scarring in the brain were also ameliorated by the administration of the agent, thereby extending the average life span even in the low dose group. Thus, arundic acid possesses not only hypotensive effects, but also prevents hypertension-derived brain damage in SHRSP.

To understand the mechanism of arundic acid, we investigated the distribution and quantity of two types of astrocyte-associated proteins, S100B and GFAP, in brain using immunohistochemistry. S100B protein has been reported to be an acidic calcium and/or zinc ion-binding soluble protein found in the cytoplasm and nucleus. S100B is predominantly expressed and secreted by astrocytes, but it is also expressed in other cells such as oligodendrocytes, ependymal cells, choroid plexus epithelial cells, vascular endothelial cells, lymphocytes, and several types of neurons in the human brain (Steiner et al. 2007; Boyes et al. 1986; Gonçalves et al. 2008). It is also reported to be expressed by astrocytes in the gray matter, oligodendrocytes, and microglia in the white matter of rodents (Gonçalves et al. 2008). Thus, expression may vary depending on the type of neuronal tissue and animal species. S100B exerts dual trophic and toxic effects, depending on its concentration (Rothermundt et al. 2003; Marenholz et al. 2004). GFAP on the other hand, is expressed in the fine fibrillary processes of reactive astrocytes (Reymond et al. 1996; Ridet et al. 1996). Thus, S100B is non-cell-specific but labels activated astrocytes, whereas GFAP is considered to be an astrocyte-specific marker protein (Ridet et al. 1996; Yasuda et al. 2004).

In this experiment, we analyzed brain tissue resected from rats following spontaneous death or euthanasia at the end of the experiment, and immunostained sections using two types of antibodies against two proteins associated with astrocytes. We analyzed the area occupied by dot and filamentous structures in control SHRSP and in SHRSP treated with arundic acid. In this study, the most specific antibodies against S100B and GFAP were used for detecting these proteins in the lesions of the brains of spontaneously hypertensive rats. If S100B is released into intercellular spaces, structures immunostained by the S100B antibody should represent filaments (Rothermundt et al. 2003; Marenholz et al. 2004; Tramontina et al. 2002). However, the total immunostained area in the cortex was larger compared with that in the white matter or hippocampus by more than $10 \%$, which contained a mixture of round particles, small dots, and circles, in addition to structures consistent with astrocytic morphology. This suggests that structures immunostained by the S100B antibody in the rat cerebral cortex may be derived from several different cell types including astrocytes, ependymal cells, vascular endothelial cells, lymphocytes, and/or several types of neurons.

S100B is a dual action protein (Rothermundt et al. 2003; Yasuda et al. 2004). At nanomolar concentrations, it stimulates neurite outgrowth and enhances survival of neurons during development of lesions. At micromolar 
levels, it stimulates expression of proinflammatory cytokines and induces apoptosis (Rothermundt et al. 2003; Petrova et al. 2000; Lam et al. 2001). How such two diverse effects are balanced remains to be elucidated. Reactive astrocytes were shown to have enhanced S100B immunoreactivity in lesions in the cerebral cortex, white matter, and pons, which are the primary areas in which stroke occurs in SHRSP.

Arundic acid administration significantly inhibited both S100B and GFAP staining. GFAP labeling, which showed spider-like thick filaments, was greatly increased in the cerebral cortex of SHRSP compared with WKY rats (Fig. 4). Since GFAP is a gliofilament produced by reactive astrocytes, GFAP-labeled structures should have a typical spider-like appearance. When the cortex of control SHRSP was compared with that of WKY brains, control SHRSP cortex was more strongly immunostained by S100B and GFAP antibodies (Fig. 4), showing activated astrocytes and inflammatory cells including ependymal cells, vascular endothelial cells, and lymphocytes. Activated astrocytes labeled with the GFAP antibody were significantly reduced in the cortex of SHRSP following the administration of arundic acid, even though immunostaining with the S100B antibody showed very few differences.

Although immunoreactive dots and filamentous structures stained by the S100B antibody were less frequently seen in the white matter compared with the cortex of SHRSP, both of increased immunostainings by the S100B and GFAP antibodies were diminished in a dose-dependent manner by the administration of arundic acid in SHRSP. These data suggest that production and release of S100B protein may have facilitated astrocyte activation and the greater expression of GFAP in SHRSP. When histological differences of astrocytes were compared with the cortex and white matter, gemistrocytic astrocytes as a protoplasmic form having a wide cytoplasm in the cortex, and astrocytes as a fibrous form in the white matter were observed as shown in Figs. 4 and 5, respectively.

In the pons of SHRSP, the findings were similar to what was seen in cortex. In the hippocampus (Fig. 6), midbrain, and cerebellum, neither S100B protein nor GFAP were significantly increased in SHRSP compared with WKY rats. The expression of these proteins was not influenced by the administration of arundic acid in SHRSP except for an effect on GFAP in the high-dose group. These findings suggest that S100B labeled both astrocytes and inflammatory cells, and that astrocytes in these areas are not readily influenced by hypertension, in contrast to cortical and white matter astrocytes.

Arundic acid is a promising drug that may prevent the occurrence of degeneration or stroke that occurs with severe hypertension. This drug may also prevent the formation of large scars, and may prevent the enlargement of stroke lesions by preventing the inflammatory changes caused by overproduction of S100B protein. We suspect that two different mechanisms are involved in the actions of arundic acid: inhibition of reactive astrocytes at a low dose and hypotensive effects at a high dose. Which of these two mechanisms is most important for tissue homeostasis is not clear at this time, but it does seem clear that arundic acid, even at a low dose, has specially protective effects on the brain, but not in the heart and kidney. Clinical trials for arundic acid are ongoing worldwide (de Paulis 2003). Our findings will contribute to the understanding of the pathophysiology of cerebral diseases with inflammation, including cerebral hemorrhage, thrombosis, embolism (Pettigrew et al. 2006a, b), Parkinson's disease, and Alzheimer's disease (Mori et al. 2006; Doraiswamy and Xiong 2006).

Acknowledgments This research was supported by the Research Funds of Kinki University School of Medicine.

Open Access This article is distributed under the terms of the Creative Commons Attribution Noncommercial License which permits any noncommercial use, distribution, and reproduction in any medium, provided the original author(s) and source are credited.

\section{References}

Boyes BE, Kim SU, Lee V, Sung SC (1986) Immunohistochemical co-localization of S-100b and the glial fibrillary acidic protein in rat brain. Neuroscience 17:857-865

de Paulis T (2003) ONO-2506. Curr Opin Investig Drugs 4:863-867

Doraiswamy PM, Xiong GL (2006) Pharmacological strategies for the prevention of Alzheimer's disease. Expert Opin Pharmacother $7: 1-10$

Gonçalves CA, Leite MC, Nardin P (2008) Biological and methodological features of the measurement of $\mathrm{S} 100 \mathrm{~B}$, a putative marker of brain injury. Clin Biochem 41:755-763

Ilg EC, Schäfer BW, Heizmann CW (1996) Expression pattern of S100 calcium-binding proteins in human tumors. Int J Cancer 68:325-332

Kataoka H, Yamada E, Hazama F (1994) Increased basic fibroblast growth factor immunoreactivity in the brain of stroke-prone spontaneously hypertensive rats. Acta Neuropathol (Berlin) 88:7-13

Lam AG, Koppal T, Akema KT, Guo L, Craft JM, Samy B, Schavocky JP, Watterson DM, van Eldik LJ (2001) Mechanism of glial activation by S100B: involvement of the transcription factor NFkappaB. Neurobiol Aging 22:765-772

Marenholz I, Heizmann CW, Fritz G (2004) S100 proteins in mouse and man: from evolution to function and pathology (including an update of the nomenclature). Biochem Biophys Res Commun 322:1111-1122

Matsui T, Mori T, Tateishi N, Kagamiishi Y, Satoh S, Katsube N, Morikawa E, Morimoto T, Ikuta F, Asano T (2002) Astrocytic activation and delayed infarct expansion after permanent focal ischemia in rats. Part I: enhanced astrocytic synthesis of S-100 $\beta$ in the periinfarct area precedes delayed infarct. J Cerebr Blood Flow Metab 22:711-722

Mori T, Tateishi N, Kagamiishi Y, Shimoda T, Satoh S, Ono S, Katsube N, Asano T (2004) Attenuation of a delayed increase in 
the extracellular glutamate level in the peri-infarct area following focal cerebral ischemia by a novel agent ONO-2506. Neurochem Int 45:381-387

Mori T, Town T, Tan J, Yada N, Horikoshi Y, Yamamoto J, Shimoda T, Kamanaka Y, Tateishi N, Asano T (2006) Arundic acid ameliorates cerebral amyloidosis and gliosis in Alzheimer transgenic mice. Pharmacol Exp Ther 318:571-578

Okamoto K, Aoki K (1963) Development of a strain of spontaneously hypertensive rats. Jpn Circ J 27:282-293

Okamoto K, Yamori Y, Nagaoka A (1974) Establishment of the stroke-prone spontaneously hypertensive rat (SHR). Circ Res 34/ 35(Suppl.I):143-153

Petrova TV, Hu J, Van Eldik LJ (2000) Modulation of glial activation by astrocyte-derived protein S100B: differential responses of astrocyte and microglial cultures. Brain Res 853:74-80

Pettigrew LC, Kasner SE, Albers GW, Gorman M, Grotta JC, Sherman DG, Funakoshi Y, Ishibashi H (2006a) Safety and tolerability of arundic acid in acute ischemic stroke. J Neurol Sci 251:50-56

Pettigrew LC, Kasner SE, Gorman M, Atkinson RP, Funakoshi Y, Ishibashi $\mathrm{H}$ (2006b) Effect of arundic acid on serum S-100beta in ischemic stroke. J Neurol Sci 251:57-61

Rettig WJ, Chesa PG, Beresfold HR, Feikert HJ, Jennings MT, Cohe J, Oettgen HF, Old LJ (1986) Differential expression of cell surface antigens and glial fibrillary acidic protein in human astrocytoma subsets. Cancer Res 46:6406-6412

Reymond I, Almarghimi K, Tappaz M (1996) Immunohistochemical localization of cysteine sulfinate decarboxylase in astrocytes in the cerebellum and hippocampus: a quantitative double immunofluorescence study with glial fibrillary acidic protein and S-100 protein. Neuroscience 75:619-633
Ridet JL, Alonso G, Chauvet N, Chapron J, Koenig J, Privat A (1996) Immunohistochemical characterization of a new marker of fibrous and reactive astrocytes. Cell Tissue Res 283:39-49

Rothermundt M, Peters M, Prehn J, Arolt V (2003) S100B in brain damage and neurodegeneration. Microsc Res Tech 60:614-632

Shimoda T, Tateishi K, Shintaku K, Shintaku K, Yada N, Katagi J, Akiyama T, Maekawa H, Shinagawa R, Kondo K (1998) ONO2506, a novel astrocyte modulating agent, suppresses the increase of COX-2 and iNOSmRNA expression in cultured astrocytes and ischemic brain. Soc Neurosci 24:384.13 (abstract)

Shinagawa R, Shimoda T, Kagamiishi Y, Kamanaka Y (2006) Role of the astrocyte-specific protein S100B in acute stroke (Article in Japanese). Nippon Yakurigaku Zasshi 127:485-488

Steiner J, Bernstein HG, Bielau H, Berndt A, Brisch R, Mawrin C, Keilhoff G, Bogerts B (2007) Evidence for a wide extra-astrocytic distribution of S100B in human brain. BMC Neurosci 8:2

Tateishi N, Mori T, Kagamiishi Y, Satoh S, Katsube N, Morikawa E, Morimoto T, Matsui T, Asano T (2002) Astrocytic activation and delayed infarct expansion after permanent focal ischemia in rats. Part II: Suppression of astrocytic activation by a novel agent (R)(-)-2-propyloctanoic acid (ONO-2506) leads to mitigation of delayed infarct expansion and early improvement of neurologic deficits. J Cerebr Blood Flow Metab 22:723-734

Tramontina F, Conte S, Gonçalves D, Gottfried C, Portela LV, Vinade L, Salbero C, Gonçalves C-A (2002) Developmental changes in $\mathrm{S} 100 \mathrm{~B}$ content in brain tissue, cerebrospinal fluid, and astrocyte cultures of rats. Cell Mol Neurobiol 22:373-378

Yasuda Y, Tateishi N, Shimoda T, Satoh S, Ogitani E, Fujita S (2004) Relationship between S100b and GFAP expression in astrocytes during infarction and glial scar formation after mild transient ischemia. Brain Res 1021:320-331 